\title{
VIA in cervical cancer screening
}

\author{
Dr. Veena Rahatgaonkar \\ Associate Professor \& In charge of Cancer detection center. Bharati Vidyapeeth University Medical College, \\ Sangli.
}

\begin{abstract}
In developing countries cancer cervix is the leading cause of cancer death in females. Effective screening contributes to decrease mortality. Lack of resources is main factor for failure of effective screening for cervical cancer. VIA, visual inspection of cervix with acetic acid application is low cost approach for cervical cancer screening in low income countries.
\end{abstract}

Key-words: Cancer, Cervix, Pap smear, Screening, VIA

\section{INTRODUCTION}

Cervical cancer is the third most common cancer in women worldwide. An estimated 500,000 new cases of cervical cancer are identified every year worldwide leading to annual mortality of 270, 000. [1] In developing countries cancer cervix is the commonest cancer \& leading cause of cancer death in females. It is the leading cause of years of life lost in women's life expectancy even when compared with AIDS, T.B., Maternal conditions in Latin America \& Europe. In India 1, 32000 new cases of cancer cervix are detected per year \& 74,000 deaths take place due to this disease each year. According to an estimate, in every two minutes a woman dies of cervical cancer \& one out of four women who die of cervical cancer is an Indian woman. [2] If disease burden goes on rising by this proportion by 2050 there will be more than 1 million new cases of cervical cancer each year in India.[3] Most of the females who die from Cancer cervix in developing countries particularly are in the prime of their life \& their death is loss to family and community. Without urgent action deaths due to cancer cervix are projected to rise by almost $25 \%$ over the next 10 years.[4]

Effective screening will contribute to lower the mortality \& morbidity associated with frank malignancy of cervix. Cytological methods of screening for cervical cancer \& precursors have become the mainstay of population based prevention programs, resulting in substantial reduction of disease in countries such as Canada \& Finland where mass screening is systematic rather than opportunistic. [5, 6] Right from the introduction of Pap smear cervical cancer is thought to be a preventable disease .The tragedy is that the disease is not yet prevented. Most of the middle \& low income countries failed to implement cervical cancer control program successfully.[7]

Lack of resources \& poorly organized health system are important factors for failure of effective screening for cervical cancer in developing countries. Hence there is need of low cost approach for effective cervical cancer screening program.[8] In 1982, Ottaviano and La Torre published a study where women who were examined visually and colposcopically after a cervical wash with acetic acid were found to have equal detection rates of cervical abnormalities by both the techniques.[9]

VIA, the method of visual inspection of cervix with acetic acid application is the preferable method for cervical cancer screening in low income countries.[10] As visual screening test does not rely upon laboratory services it is the most promising alternative to cytology where resources are limited.[11,12]

\section{Selection Of Women For Via}

VIA is indicated for women for whom cervical cancer screening is recommended \& for whom this method is the best screening option where access to cervical cytology and HPV testing is not available. VIA can be done in women of all age groups after the onset of sexual activity up to menopause. It is not suitable for postmenopausal women. In low income countries when resources are limited, higher priority for screening is given to women aged 35 years or more and repeat screening in every 5 years will achieve great impact on incidenceand mortality associated with cervical cancer.

\section{Contraindications For Via}

There are no contra indications to perform VIA. It can be done during pregnancy.

\section{Technique Of Via}

Equipments required for the test are not costly \& are as follows:

- Examination table

- A bright light source preferably, Halogen lamp 
- $\quad$ Sterile gloves

- Cusco's speculum

- 3-5\% Acetic acid

- Cotton tipped swabs

- Recording forms

Before carrying out the procedure, informed consent of the woman is taken. Relevant Obstetric and Gynecological history should be obtained. Vulva is examined for any warts, ulcer or infections. Cusco's speculum is inserted \& inspection of cervix is done. The color, amount and smell of cervical discharge are noted.

Light is adjusted in order to get the best view of cervix. Any discharge, blood or mucus from cervix is removed with help of cotton swabs. $5 \%$ acetic acid is applied to cervix. One minute after application of acetic acid cervix is examined for development of any acetowhite area near the squamocolumnar junction. Acetic acid coagulates nuclear proteins. Hence when there is more nuclear material in proliferating cells of dysplastic epithelium, acetic acid coagulates these proteins obliterating the color of the stroma and the abnormal areas appear as acetowhite areas. The intensity of acetowhiteness, borders of acetowhite area \& location of acetowhite area are noted.[13]

After the test, findings are noted on recording form. Abnormal findings can bedocumented by diagrammatic representation.

\section{Reporting Of Outcome Of Via Test}

The test results are categorized into the following three groups:

1) VIA negative

2) VIA positive

3) VIA positive for cancer

If there is no Aceto white area on the cervix the test is negative. Ill defined, patchy, pale, discontinuous, scattered acetowhite areas away from squamocolumnar junction are also considered as negative. (fig.1)

When there is distinct, well defined, dense acetowhite area close to or abutting the squamocolumnar junction the test is considered as positive. (fig. 2) In case of VIA negative report, the woman is called after 5 years for rescreening.

In case of VIA positive report, woman should be referred for further investigations such as Colposcopy and colposcopy guided biopsy and treated as per any confirmed lesions. VIA positive case can also be treated immediately using conservative approach like cryotherpy, loop electrosurgical excision procedure (LEEP) as in single visit approach. With sufficient skills, 10 to $20 \%$ of women examined may be scored as VIA positive and one or more of five VIA positive outcomes result in diagnosis of CIN of any grade.

\section{VI. $\quad$ Efficacy Of Via}

Sensitivity of VIA for detection of precancerous \& cancerous lesions is in range of $56 \%$ to $96 \%$ average is $77 \%$ \& Specificity ranges from 74 to $94 \%$ with an average of $86 \%$.

A range of multi -centric cross sectional studies conducted by IARC \& WHO in India \& Africa in the recent past have proved VIA performed by trained paramedics has higher test accuracy compared to Pap smear performed in same settings. [Sensitivity of VIA 77\%(58\%- 94\%) \& sensitivity of Cytology 58\%(29\%-77\%)]

The comparative efficacy of visual inspection with acetic acid, HPV testing (Hybrid Capture II) and conventional cytology in cervical cancer screening was evaluated in a randomized intervention trial in Maharashtra, India. The study observed that the detection rates of CIN 2 and worse lesions were similar with all the methods. In contrast to the other laboratory based tests, VIA provided immediate results based on which colposcopy and management could be done at the same visit, making the test very cost-effective.[14]

Ghaemmaghami tested VIA for cervical cancer screening in Iran where cervical cancer is the leading cause of cancer related death in women .1,200 women between the ages 18 and 70 from Teharan ,Iran were screened by VIA \& Pap Smear .The sensitivity of VIA was found to be $74.3 \%$ compared with $72 \%$ of Pap smear. The specificity of VIA was $94 \%$ compared to $90.2 \%$ of Pap smear. Ghaemmaghami concluded that sensitivity \& specificity of VIA is high comparable with that of cytology making it feasible method of screening in countries where access to cytopathology is limited.[15]

In rural areas of Northeast Brazil, Bomfirm studied 1,154 women who were screened by VIA \& Pap smear done by physicians or nurses. Sensitivity of VIA was found to be $100 \%$ while $18 \%$ for Pap smear \& specificity was $78 \%$ for VIA vs. $100 \%$ for Pap smear in detecting LSIL \& HSIL. The positive predictive value of VIA was $15.6 \%$ for LSIL and $2.8 \%$ for HSIL. Negative predictive value of VIA is $100 \%$ for both type of lesions \& for Pap smear the negative predictive value is $97 \%$ \& $99 \%$. Bomfirm concluded that VIA could be an excellent screening method for detecting cervical cancer.[16]

In urban Africa, Nairobi (Kenya) De Vuyst studied VIA comparing it to three other methods, that is Pap smear, HPV DNA testing \& cervicography in 653 women. The gold standard for diagnosis was colposcopy 
\& biopsy. Sensitivity \& specificity were $83.3 \%$ \& $94.6 \%$ for Pap smear, $73.3 \%$ \& $80 \%$ for VIA, $94.4 \%$ and 73.9\%HPV PCR \& $72.3 \%$ \& 93.2\% for Cervicography. Although Pap smear had the highest sensitivity \& HPV PCR highest specificity, VIA showed the accuracy in between these two tests. So he concluded that in low resource countries VIA is effective as primary screening tool.[17]

In 2003-2004, concurrent studies were performed by the Alliance for cervical cancer prevention, across the world, looking at VIA as an alternative to Pap smear for cervical cancer screening. Studies were conducted in countries with major burden of disease like India, Africa and Central America. Doh examined VIA as screening method in Cameroon, Africa. 4,813 women were screened .Sensitivity of VIA was $70.4 \%$ vs. $47.7 \%$ for Pap .VIA specificity was $77.6 \%$ vs. $94.2 \%$ for Pap smear .Positive predictive value of VIA $44 \%$ and negative predictive value is $91.3 \%$.Doe concluded that although Pap has slightly better testing qualities, VIA has acceptable test qualities and may be used as large scale screening method in low resource settings. [18]

A cluster randomized trial conducted in Tamil Nadu, India aimed to evaluate the effect of visual screening on cervical cancer incidence and mortality after a single round of VIA screening followed by cryotherapy. Follow up of the entire cohort after 7 years showed a significant 25 percent reduction in cervical cancer incidence (hazard ratio 0.75 ), a significant 35 percent reduction in cervical cancer mortality (hazard ratio 0.65 ) and a 27 percent reduction in the incidence of stage II or advanced cancers (Hazard Ration 0.73) in the intervention group as compared with control group. This study indicates that VIA screening, in the presence of good training \& sustained quality assurance is an effective method to prevent cervical cancer in developing countries. [19]

\section{Advantages Of Via}

- This test is relatively simple, no specialized skills are required. It can be taught to nurses, health workers \& paramedical staff. Requires a training of 5-14 days only. So it can be used even at rural hospitals, in camps at periphery for screening. To maintain quality services it is important that an experienced provider should conduct regular assessment.

- Sensitivity of VIA in detecting high grade lesions seems to be as good as cytology.

- It does not cause discomfort to women as the procedure is painless \& requires only 2-3 minutes.

- VIA is less costly than other screening tests in routine use \& minimal infrastructure is required for it.

- It can be used where well equipped labs \& cytopathologists are not available for interpretation of Pap smear slides

- No transport or laboratory equipment or personnel required

- VIA is real time screening test. A result of the test is available immediately, eliminating need for second visit for collection of report \& reducing loss to follow up cases.

- VIA could be potentially be used in an approach based on screening \& treating women in single visit. Recall of women may not be necessary, there by potentially minimizing default rates for treatment of women with positive screening tests.

In 2005, after many evidence based articles substantiated VIA as an adequate screening method for cervical cancer, Lawrence went to Guatemala and implemented a pilot study in evaluating VIA with "See and Treat method". Lawrence looked at the acceptability of cervical screening using direct visual inspection after acetic acid application followed by immediate cryotherapy for CIN among women in rural Guatemala. Lawrence and colleagues offered cervical cancer screening to 1,052 women using VIA.9.3\%of women deferred screening at all and refused examination. Among the 954 women screened, $13 \%$ were found to have findings consistent with CIN I or higher $.99 \%$ of the women with positive findings agreed to undergo immediate treatment with cryotherapy. This study shows that VIA with "See and Treat" method could be accepted by patients in developing countries.[20]

\section{Limitations}

- VIA cannot be relied on in postmenopausal women as the squamocolumnar junction recedes in endocervical canal. Test has inherent difficulties in identifying endocervical disease.

- VIA has low specificity compared with cytology and has high rate of false positivity. Adequate training of health workers is important to reduce falls positive referrals.

- Positive predictive value of the test is less, so considerable numbers of women with positive test do not have the disease resulting in overdiagnosis \& treatment. There is chance of high referral rates which causes unnecessary anxiety in women.

- There is no permanent record of the test that can be reviewed later.

- There is lack of standardization .The test has inherent limitation of subjective variation. No standardized method of quality control is available.

- Training methods and competency evaluation are not yet standardized. 


\section{Current Scenarios Regarding Via}

VIA is currently being tested in large cross sectional, randomized controlled trials in developing countries. Limited evidence is available as to determine effect on incidence of cervical cancer \& mortality. Whether VIA works in an integrated service delivery model with other competing demands for provider time, knowledge and skills is not yet proven as most of the data is from the research setting. VIA is recommended as a screening method in closely monitored settings. This method should not be recommended for postmenopausal women.

Recent data indicates that VIA is as effective as Pap smears in detecting the disease \& has less technical constraints. The non invasive nature, easy applicability \& low cost of the test make VIA a feasible screening method in low income countries.

In Zimbabwe, in collaborative project of university of Zimbabwe \& JHPIEGO Cervical cancer project 1999, midwives easily learned to perform VIA screening at primary health center after a week competency based training course \& could identify women with no disease \& those required referral for further evaluation. The participants used specially designed VIA cervical atlas \& practiced VIA on models before working with patients.[21]

IARC conducted several studies in different developing countries to establish usefulness of VIA for screening of cervical cancer. Based on these studies; ACOG, the Canadian society of Gynecologist, The international Federation of Gynecology \& Obstetrics(FIGO) have all endorsed VIA as a viable option for screening in low resource settings.

\section{Conclusion}

VIA is simple, cost effective approach for cervical cancer screening. It does not require sophisticated equipment and can be done by trained health workers. The results of the test are available immediately making it an attractive approach for use in low resource settings. In India, National cancer control program recommended providing VIA based screening at the primary health centers by the trained nurses, health workers and then a single visit approach for colposcopy and management at the district hospital. [22] As VIA is an entirely provider dependent screening method the training and periodic retraining are most important determinants of good performance of VIA by providers

\section{References}

[1] Feriay J. et.al.,Cancer incidence, mortality, prevalence worldwide (IARC cancer base ,Lyon,2004)

[2] WHO Summary report on HPV\& cervical cancer statistics in India (18/03/2008)

[3] Parkin DM, et al Eur. J. Cancer 2001, 37 (Suppl. 8) 54-56

[4] Comprehensive Cervical Cancer Control, A guide to essential practice, WHO (Practice sheet 10 : Visual screening methods 123 124)

[5] Lewis M. Situational analysis of cervical cancer in Latin America and the Caribbean. Washington, DC: Pan American Health Organization;2004

[6] Cervix cancer screening .IARC handbooks of cancer prevention, vol.10. Lyon, France:IARC Press;2004

[7] ACCP: visual screening approaches (Cervical cancer prevention fact sheet (Oct 2000)

[8] Denny L., Kuhn L., Pollack A., Wainwright H, Wright T.C., Jr.(2000)Evaluation of alternative methods of cervical cancer screening for resource poor settings.Cancer,89,826-833

[9] Ottaviano M, La Torre P. Examination of the cervix with the naked eye using acetic acid test. Am J Obstet Gynecol 1982; 143:139142.

[10] Goldie S.J., Kuhn L., Denny L., Pollack A., Wright T.C. (2001) Policy analysis of cervical cancer screening strategies in low resource settings: clinical benefits and cost effectiveness. JAMA,285,3107-3115

[11] Sankaranarayanan R., Shyamalakumary B., Wesley R., Sreedevi Amma N., Parkin D.M., Krishnan Nair M.(1999) Visual inspection with acetic acid in the early detection of cervical cancer and recursors. Int. J. Cancer, 80,161-163

[12] Sankaranaryanan R., Budukh A.M., Rajkumar R. (2001) Effective screening programmes for cervical cancer in low and middle income developing countries. Bull. World Health Org.,79,954-962

[13] R. Sankarnanaryanan, Ramani S. Wesley,Testing and reporting the results of visual inspection with 5\% acetic acid (VIA), A practical manual on visual Screening for cervical Neoplasia,18-25.

[14] Sankaranarayanan R., Nene BN, Dinshaw KA, Mahe C, Jayant K, Shastri, Chinoy R, Kelkar R, udukh AM, Keskar V, Rajeshwarker R, Muwonge R, Kane S, Parkin DM A cluster randomized controlled trial of visual, cytology and HPV screening for cancer of the cervix in rural India. Int J Cancer 2005; 116: 617-623.

[15] Gaffikin L, Lautebach M, Blumenthal PD., Performance of VIA for cervical cancer screening: A qualitative summary of evidence to date, Obstetrics \& Gynecological review58(8):543-550(August 2003)

[16] Bomfim S."Visual inspection with acetic acid for cervical cancer detection”, International Journal of Gynecology \& Obstetrics, 88, 2005.

[17] De Vuyst H., Comparison of Pap smear, visual inspection with acetic acid, HPV DNA PCR testing and cervicography; International journal of Gynecology \& Obstetrics89; 2005

[18] Doh A.S.Visual inspection with Acetic acid and cytology as screening methods for cervical lesions in Cameroon, International journal of Gynecology and Obstetrics 89, 2005.

[19] Sankaranarayanan R., Esmy PO, Rajkumar R, Muwonge R, Swaminathan R, Shanthakumari S, Fayette JM, Cherian J.,Effect of visual screening on cervical cancer incidence and mortality in Tamil Nadu, India: a cluster-randomized trial, Lancet 2007; 370:398406.

[20] Lawrence MJ, Wigton TR, Leonhardt JG. Screening for cervical neoplasia in an unselected Rural Guatemalan population using direct visual inspection after acetic acid application, A pilot study, Journal of lower genital tract disease 9(4)232-235, 2005. 
[21] Royal Thai College of Obstetricians and Gynecologists (RTCOG) and the JHPIEGO Corporation cervical cancer prevention group (2003) Safety, acceptability, feasibility of a single visit approach to cervical cancer prevention in rural Thailand: A demonstration project. Lancet, 361, 814-820.

[22] Guidelines for cervical cancer screening program, National cancer control program, Govt. of India-World health organization collaborative program,

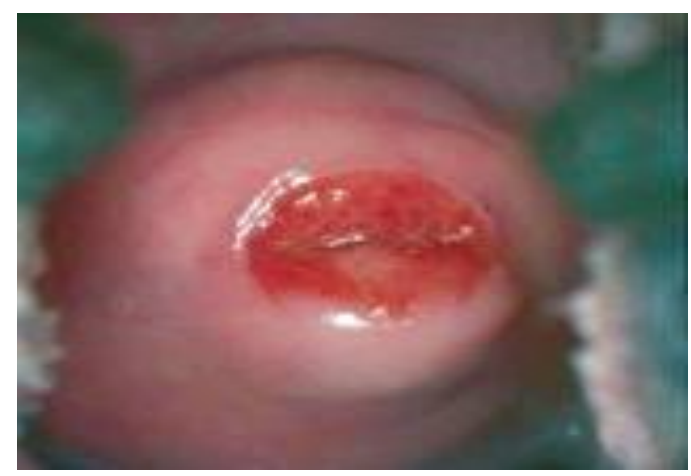

fig. 1 - via negative

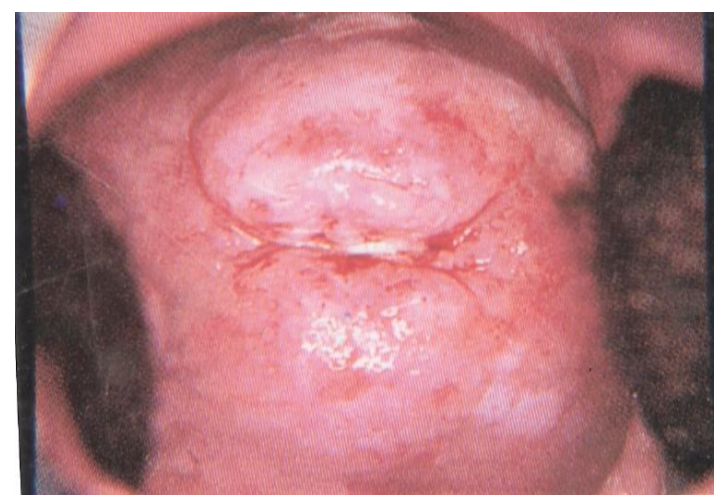

fig. 2 - via positive 